\title{
Improving the effectiveness of self-monitoring of blood glucose in type 2 diabetes
}

Around 6 years ago self-monitoring of blood glucose (SMBG) was identified as an area of costly, often inappropriate prescribing by commissioning bodies. Unnecessary prescribing of testing strips appeared easy to identify using simple searches on practice computers. In this issue of the BJGP, Robson et al describe a study of an intervention using a multimodal strategy including guideline development and implementation, education, support of clinicians and patients, and feedback on performance. This was designed to tackle the issue in the Tower Hamlets, and City and Hackney Clinical Commissioning Groups (CCGs), with Newham CCG serving as a control. ${ }^{1}$ They demonstrate that in this region, the intervention (which promoted case by case review by clinicians as well as computer searches) substantially reduced the prescribing of SMBG strips compared to the control.

Both CCG policy and the Robson study were motivated by a lack of evidence supporting SMBG in large numbers of patients with type 2 diabetes not using insulin. Numerous randomised trials have measured potential benefits in this group, particularly improvements in $\mathrm{HbA1c}$ and psychological outcomes. Despite a common assumption of 20 years ago that glycaemic control and quality of life would be improved, these trials suggest otherwise. The most recent Cochrane Review reports that for patients with type 2 diabetes not taking insulin there is little, if any, improvement in $\mathrm{HbA1c}$, and no effect on patient satisfaction, general wellbeing or general health-related quality of life.?

\section{IMPROVED DETECTION OF HYPOGLYCAEMIA}

However, a separate question concerns the ability of self-monitoring to improve safety by reducing risk of hypoglycaemia and its complications, particularly among drivers. This is much more difficult to investigate. Mild hypoglycaemia is more, not less frequently recorded in those who self-monitor, because regular selfmonitoring makes the patient more aware of hypoglycaemic episodes. ${ }^{2}$ More severe events that could cause an accident are relatively rare. So while SMBG has not been shown to reduce rates of hypoglycaemia, it improves detection. Partly for this reason, policy has moved towards wider provision

while SMBG has not been shown to reduce rates of hypoglycaemia, it improves detection.

of SMBG in patients with type 2 diabetes not using insulin. This shift is enshrined in recommendations published by the Driver and Vehicle Licensing Agency (DVLA) ${ }^{3}$ and by Diabetes UK. ${ }^{4}$

All people taking insulin should selfmonitor, and as the authors state, for those taking oral drugs that raise the risk of hypoglycaemia the DVLA advises that:

'It may be appropriate to monitor blood glucose ldepending on clinical factors including frequency of driving) at times relevant to driving to enable the detection of hypoglycaemia.

\section{ORAL DRUGS CAUSING HYPO RISK}

Which are the oral drugs associated with risk of hypoglycaemia? They are the sulphonylureas and the meglitinides. These two groups act by promoting insulin secretion, actively driving blood glucose levels down, potentially too far at times. The sulphonylureas are a very commonly used drug class that is becoming displaced by newer drugs that do not carry this risk. This threatens a reversal of the costsaving initiatives of recent years, as we now have more therapeutic options that carry very little risk of hypoglycaemia unless co-prescribed with drugs that do. So a driver taking a sulphonylurea could either be offered SMBG or a change to a more expensive newer agent without the need for it. The meglitinides (repaglinide and nateglinide) are less commonly used than sulphonylureas but are also inexpensive and have the advantage that their duration of action is shorter, reducing, but not eliminating, the associated risk of hypoglycaemia.

Interestingly, none of the 12 studies included in the Cochrane Review ${ }^{2}$ were designed to investigate specifically the subgroup at risk of hypoglycaemia through oral medication. In fact, eight of the 12 do not report the numbers receiving such drug therapy, suggesting a lack of recognition at the time of the relevance of this factor.
Of the four that do, usage of such drugs in the trial populations varied from $3.2 \%{ }^{5}$ to $80.8 \%,{ }^{6}$ representing significant clinical heterogeneity. One study, DiGEM, ${ }^{7}$ identified a pre-specified subgroup based on type of therapy land was powered to detect benefit in it), but this was any oral hypoglycaemic drug therapy versus dietary management alone. Only in quite recent times have oral drugs associated with hypoglycaemia risk been identified specifically as a potential justification for SMBG, except in one particular scenario where this is well established: fasting during Ramadan. Hypoglycaemia is a subtle phenomenon in its milder forms, and while most patients are aware of it la requirement for a driving licence), the symptoms overlap with other experiences, including simple hunger. This is particularly relevant to those choosing to fast, and in such people the provision of self-monitoring is recommended during this period, ${ }^{8}$ particularly in those taking medication associated with risk of hypoglycaemia. ${ }^{9}$

\section{NEED FOR INDIVIDUALISED DECISIONS}

Sulphonylureas are usually very effective at reducing blood glucose levels quickly, but an individual's responsiveness is variable and difficult to predict. Occasionally patients need to start a sulphonylurea to control blood glucose levels destabilised through inter-current infection, when a short-term need for SMBG may arise. This may similarly occur in the symptomatically hyperglycaemic patient requiring rapid control through sulphonylurea dose titration. Systemic corticosteroids can also affect blood glucose in an unpredictable way and may again justify a decision to offer SMBG on a short-term basis. The key is that the decisions should be individualised and SMBG should be linked to structured follow-up and care.

Five years ago the assumption was that SMBG prescribing in non-insulin users was inappropriate in all but a negligibly small minority, and this assumption is reflected 


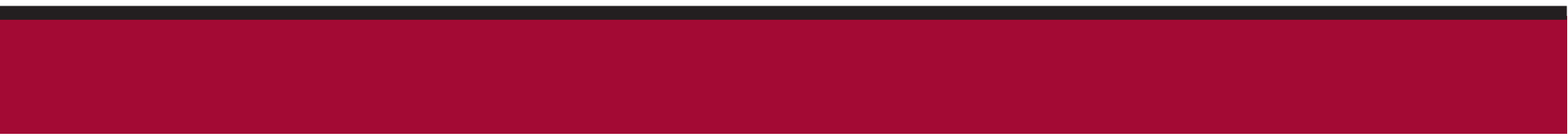

"... it is important for both clinical and medicolegal reasons that a GP in today's world does not automatically decline a request for SMBG testing simply because the patient is not using insulin.

\section{ADDRESS FOR CORRESPONDENCE}

Tim A Holt

University of Oxford, Nuffield Department of Primary

Care Health Sciences, Radcliffe Observatory

Quarter, Woodstock Road, Oxford OX2 6GG, UK.

E-mail: tim.holtaphc.ox.ac.uk in the Robson et al study's main outcome measure. But how small in fact is this 'minority' that may benefit? As mentioned above, in the study populations of the Cochrane Review 3.2\% to $80.8 \%$ were taking a sulphonylurea. We do not know exactly how many of these hold a driving licence lor operate machineryl, how many fast during Ramadan, or how many have other, individual reasons that may justify SMBG. Some patients may of course belong to all three groups at once. So it is important for both clinical and medicolegal reasons that a GP in today's world does not automatically decline a request for SMBG testing simply because the patient is not using insulin.

The people who ask us for repeat prescriptions for SMBG strips because they believe they personally benefit are a different population to those enrolled in randomised controlled trials of SMBG, most of whom are naive to this practice. We should also note that the 2012 Cochrane Review² did not confirm the previous evidence up to 2009, suggesting that SMBG is actively harmful in people not on insulin, evidence cited by Robson et al as part of the basis for their study.

Looking at it more positively, many patients can be identified in the spirit of Robson et al's intervention, who may well be labouring under the yoke of frequent SMBG to no benefit, and costing the NHS considerable sums in the process. Such individuals may indeed be liberated by stopping this practice. It is not too difficult to identify some such people lthose not using a sulphonylurea, meglitinide, or insulin). The outcomes reported by Robson et al related to those only taking metformin lor no drug therapyl are therefore more valid than the other comparisons.

\section{AVOIDING BLANKET DECISION MAKING}

GPs have to keep up to date with changing policy in all areas of care, and are inevitably vulnerable to over-simplifications, particularly when presented as headline statements. So the only message here is that decision making over prescribing SMBG, and frequency of testing should be individualised and patient centred. SMBG should not be started routinely in all patients and always needs to be justified as part of a structured programme of care. Many people self-monitor inappropriately and there is scope to improve care and save money by stopping it in those with no risk of hypoglycaemia. But this can only be achieved safely if the determinants of hypoglycaemia risk and its complications lcurrent drug therapy, driving status, fasting behaviour, systemic corticosteroids, need for short-term dose titration) are fully taken into account.

Finally, we live in an age where (rightly or wronglyl, self-monitoring of all sorts of physiological parameters is becoming increasingly prevalent. It is possible that failure so far for SMBG to substantially improve $\mathrm{HbA1c}$ in randomised trials of patients with type 2 diabetes not using insulin is related to the way patients are taught to interpret and respond behaviourally to their results. Progress in this area may in future provide a more satisfactory justification for SMBG (based on improved $\mathrm{HbA} 1 \mathrm{c}$ ) in a more targeted type 2 population. This was one of the conclusions of a further meta-analysis in this area of care. ${ }^{10}$ In the meantime, a new guideline from the National Institute for Health and Care Excellence for type 2 diabetes will be published this summer, and will clarify the role of newer agents and of self-monitoring of blood glucose in current practice.

\section{Tim A Holt,}

Senior Clinical Research Fellow, Nuffield

Department of Primary Care Health Sciences, University of Oxford, Oxford.

\section{Provenance}

Commissioned; not externally peer reviewed.

DOI: 10.3399/bjgp15X684277

\section{REFERENCES}

1. Robson J, Smithers H, Chowdhury T, et al. Reduction in self-monitoring of blood glucose in type 2 diabetes: an observational controlled study in inner London. $\mathrm{Br} \mathrm{J}$ Gen Pract 2015; DOI: 10.3399/bjgp15X684421.

2. Malanda UL Welschen LMC, Riphagen II, et al. Self-monitoring of blood glucose in patients with type 2 diabetes mellitus who are not using insulin (Review). Cochrane Database Syst Rev 2012; 1: CD005060.

3. Driver and Vehicle Licensing Agency. For medical practitioners. At a glance guide to the current medical standards of fitness to drive. 2014. https://uww.gov.uk/government/ publications/at-a-glance laccessed 16 Feb 2015).

4. Diabetes UK. Position statement. Self monitoring of blood glucose (SMBG) for adults with Type 2 diabetes. 2013. http://wnw. diabetes.org.uk/Documents/Position\%20 statements/Diabetes-UK-position-statementSMBG-Type2-0413.pdf (accessed 16 Feb 2015).

5. Franciosi M, Lucisano G, Pellegrini F, et al. ROSES: role of self-monitoring of blood glucose and intensive education in patients with Type 2 diabetes not receiving insulin. A pilot randomized clinical trial. Diabet Med 2011; 28(7): 789-796.

6. Guerci B, Drouin P, Grange V, et al. Selfmonitoring of blood glucose significantly improves metabolic control in patients with type 2 diabetes mellitus: the Auto-Surveillance Intervention Active (ASIA) study. Diabetes Metab 2003; 29(6): 587-594.

7. Farmer A, Wade A, Goyder E, et al. Impact of self monitoring of blood glucose in the management of patients with non-insulin treated diabetes: open parallel group randomised trial. BMJ 2007; 335(7611): 132.

8. Hui E, Bravis V, Hassanein M, et al. Management of people with diabetes wanting to fast during Ramadan. BMJ 2010; 340: c3053.

9. Al-Arouj M, Assaad-Khalil S, Buse J, et al. American Diabetes Association commentary: recommendations for management of diabetes during Ramadan. Update 2010. Diabetes Care 2010; 33(8): 1895-1902.

10. Farmer AJ Perera R, Ward A, et al Meta-analysis of individual patient data in randomised trials of self monitoring of blood glucose in people with non-insulin treated type 2 diabetes. BMJ 2012; 344: e486. 